\title{
Rapid thermal processing of self-assembling block copolymer thin films
}

\author{
F Ferrarese Lupi, ${ }^{* a}$ T J Giammaria, ${ }^{a, b}$ M Ceresoli, ${ }^{a, c}$ G Seguini, ${ }^{a}$ K Sparnacci, ${ }^{b}$ D Antonioli, ${ }^{b}$ \\ V Gianotti, ${ }^{\mathrm{b}}$ M Laus ${ }^{\mathrm{b}}$ and M Perego ${ }^{\mathrm{a}}$
}

\author{
a Laboratorio MDM, IMM-CNR, Via C. Olivetti 2, 20864 Agrate Brianza (MB), Italy \\ ${ }^{\mathrm{b}}$ Dipartimento di Scienze e Innovazione Tecnologica (DISIT), Viale T. Michel 11, Università del \\ Piemonte Orientale “A. Avogadro”, INSTM, UdR Alessandria, 15121 Alessandria, Italy \\ ${ }^{\mathrm{c}}$ Dipartimento di Fisica, Università di Milano, Via Celoria 16, I-20133 Milano, Italy
}

e-mail: federico.ferrareselupi@mdm.imm.cnr.it

\begin{abstract}
Self-assembling block copolymers generate nanostructured patterns which are useful for a wide range of applications. In this paper we demonstrate the capability to control the morphology of the self-assembling process of PS-b-PMMA diblock copolymer (DBC) thin films on un-patterned surfaces by means of fast thermal treatment performed in a Rapid Thermal Processing (RTP) machine. The methodology involves the use of radiation sources in order to rapidly drive the polymeric film above the glass transition temperature. Highly ordered patterns were obtained for perpendicular-oriented cylindrical and lamellar PS-b-PMMA block copolymers in less than $60 \mathrm{~s}$. This approach offers the unprecedented opportunity to investigate in details the kinetics of the block copolymer self-assembly during the early stages of the process, providing a much deeper understanding of the chemical and physical phenomena governing these processes.
\end{abstract}

\section{Introduction}

Technologies based on self-assembling (SA) materials, as diblock copolymers (DBC), are gaining increasing interest in microelectronics, representing viable bottom-up options to beat the limitations of current top-down lithographic processes for the fabrication of sub-20 nm features. ${ }^{[1-3]}$ Such bottom-up approaches could enable to overcome the $\lambda / 4$ resolution limit of the traditional $193 \mathrm{~nm}$ photolithographic technique as well as the high costs and time constrains of multiple exposure procedures $^{[4]}$ involved in alternative radiation-based lithographic techniques applied on very large scale (VLS) manufacturing. ${ }^{[5-8]}$ The possibility of integrating SA materials in standard lithographic 
processes, to generate sub-lithographic structures, was critically reviewed in recent editions of the Semiconductor Industry Association's International Technology Roadmap for Semiconductors (ITRS) in the perspective of process simplification. ${ }^{[9]}$ In this frame, polystyrene-bpolymethylmethacrylate (PS-b-PMMA) revealed one of the most promising SA material.

The standard fabrication procedure to prepare a nanostructured polymeric layer involves essentially two steps, the substrate surface neutralization and the DBC ordering. ${ }^{[10]}$ In the first step, the surface neutralization, i.e. the non-preferential wettability of the substrate respect to the PS and PMMA blocks, can be obtained through a thermally-induced grafting reaction of the substrate with a $\mathrm{OH}$ terminated random polystyrene-r-polymethylmethacrylate (PS-r-PMMA) copolymer (RCP). The second step consists of the deposition of the DBC layer and subsequent annealing treatment, to promote perpendicular orientation of the cylindrical or lamellar microphase separated morphologies. Although sub-20 nm patterns, with density approaching 1 terabit/inch ${ }^{2}$, were obtained, ${ }^{[11,12]}$ the technological implementation of the above procedure is precluded by the excessively long duration of the thermal treatments. Considering both steps, all the standard thermal procedures reported so far require annealing on a time scale comprised between $10^{2}$ and $10^{3} \min$. [12-14] This crucial issue is specifically addressed in the ITRS. To be competitive with the actual large-scale production lithographic techniques, the net time to form and fix the DBC pattern must lay around 240 seconds. ${ }^{[15]}$

Taking into account the guidelines defined by the ITRS, significant progresses have been reported in the literature leading to a remarkable scaling down of the annealing time required by the DBC to self-organize. This task has been accomplished through different techniques, such as solvent, ${ }^{[16-18]}$ microwave, ${ }^{[19]}$ or thermal ${ }^{[20]}$ annealing, directly acting on the kinetic of the system in order to increase the DBC mobility. Among these approaches, the solvent annealing treatment represents the most investigated solution since it allows the DBC organization to be obtained in less than $600 \mathrm{~s}$ at very low temperatures. ${ }^{[18]}$ These results can be further improved introducing the microwave annealing process, as an extension of the solvent-driven process. ${ }^{[19]}$ Nevertheless, the main drawback of the aforementioned techniques is the complexity of the solvothermal setup. Moreover, the necessity to finely control all the process parameters (pressure of the chamber, solvent temperature, sample temperature, etc.) prevents a feasible application on VLS production. Similarly, short processing times $(t=60 \mathrm{~s})$ were obtained by means of thermal processes performed on hot plates. ${ }^{[21]}$ However a systematic study of the behavior of the morphology of DBC thin films in the very early stages of their evolution (few seconds after the heating) has never been reported so far, due to the impossibility of fine-tuning the rate of the total thermal energy transferred to the sample. In this regard the main limitation is the mechanism of heating transfer to the sample. The 
transfer rate is determined by the thermal conduction properties of the specific substrate under investigation.

In this contest, we propose a novel technological approach able to overcome those limitations and to pave the way to the study and the comprehension of the early stages of the SA process. This technology is based on the annealing of the deposited DBC layer in a rapid thermal processing (RTP) machine, that is a standard semiconductor manufacturing equipment. Respect to the treatments performed on the hotplate or in a conventional furnace, in which the heating transfer is governed by conduction or convection mechanisms, the radiative energy sources (normally halogen lamps) of the RTP provide several advantages. First of all the in the RTP the temperature on the sample can be tuned with extremely high heating rates (up to $50^{\circ} \mathrm{C} / \mathrm{s}$ ) compared to that of the conventional furnaces $\left(3-5^{\circ} \mathrm{C} / \mathrm{min}\right)$. In situ monitoring of the temperature induced on the sample is possible and the behavior of the temperature can be controlled during all the three phases of the annealing process: the heat up ramp, the steady state and the cooling process. For those reasons the annealing in the RTP can be performed at temperatures just below the order-disorder transition (ODT) temperature $\left(\mathrm{T}_{\text {odt }}\right)$ for very short time periods thus minimizing the effect of degradation on the thin film of both the RCP and DBC while reducing the viscosity of the polymeric film. In addition, the fast cooling rate should allow non-equilibrium morphologies to be efficiently frozen below the glass transition temperature $\left(\mathrm{T}_{\mathrm{g}}\right)$.

\section{Experimental details}

\subsection{Sample Preparation}

Oriented silicon (100) substrates with a $50 \mathrm{~nm}$ thick thermal silicon dioxide layer were used as a support for the block copolymer SA. The oxidized substrates (about $1 \mathrm{~cm}^{2}$ surface) were cleaned with Piranha solution $\left(\mathrm{H}_{2} \mathrm{SO}_{4} / \mathrm{H}_{2} \mathrm{O}_{2}\right.$ with $3 / 1$ vol. ratio at $80^{\circ} \mathrm{C}$ for $\left.40 \mathrm{~min}\right)$ to eliminate any residual organic material and to increase the surface concentration of hydroxyl groups. The samples were then rinsed in $\mathrm{H}_{2} \mathrm{O}$ and dried under $\mathrm{N}_{2}$ flow. After the cleaning process, a solution of PS-r-PMMA (18 $\mathrm{mg}$ in $2 \mathrm{ml}$ of toluene) was prepared using an ultrasonic bath and then spun on the substrates for $30 \mathrm{~s}$ at $3000 \mathrm{rpm}$. In the case of cylinders, a PS-r-PMMA with styrene fraction $0.62, \mathrm{M}_{\mathrm{n}}=13500$ $\mathrm{g} \cdot \mathrm{mol}^{-1}$ and PDI $=1.26$ (Polymer Source) was employed. For the lamellae, a PS-r-PMMA with styrene fraction $0.58, \mathrm{M}_{\mathrm{n}}=11400 \mathrm{~g} \cdot \mathrm{mol}^{-1}$ and PDI $=1.64$ (Polymer Source) was used. The samples were then thermally treated, to promote the grafting reaction. In both cases, the non-grafted PS-rPMMA fraction was removed by washing the samples with toluene using an ultrasonic bath.

Two different block copolymers were employed: the asymmetric PS-b-PMMA with styrene unit fraction 0.71, average number molecular weight $\mathrm{M}_{\mathrm{n}}=67100 \mathrm{~g} \cdot \mathrm{mol}^{-1}$, and PDI $=1.09$ (Polymer 
Source) and the symmetric PS-b-PMMA with styrene unit fraction $0.50, \mathrm{M}_{\mathrm{n}}=51000 \mathrm{~g} \cdot \mathrm{mol}^{-1}$, and PDI $=1.06$ (Polymer Source). The thermal treatments on both copolymers were performed at 190 and $250^{\circ} \mathrm{C}$. The lower temperature was chosen equal to the one of the standard furnace process ${ }^{[10]}$ for comparison purposes, whereas the effect of a substantially higher annealing temperature was investigated to provide a significant reduction of the melt viscosity and consequently of the assembling time. $^{[21]}$

Films of controlled thickness (about $28 \mathrm{~nm}$ ) of both PS-b-PMMA copolymers were deposited on the properly neutralized substrates via spin-coating from dilute solutions in toluene $(18 \mathrm{mg}$ in $2 \mathrm{ml}$ for the PS-b-PMMA with styrene unit fraction 0.71 , and $16 \mathrm{mg}$ in $2 \mathrm{ml}$ for the PS-b-PMMA with styrene unit fraction 0.50) employing the previously described procedure. The thickness of the polymeric films was measured by means of a M-200U spectroscopic ellipsometer (J. A. Wollam Co. Inc.) using a Xenon laser lamp at $70^{\circ}$ incident angle.

The opening of the cylindrical nano-template was completed by selective removal of the PMMA blocks. The degradation of the PMMA component via chain scission was achieved by exposing the samples to UV radiation $\left(5 \mathrm{~mW} \cdot \mathrm{cm}^{-2}, \lambda=253.7 \mathrm{~nm}, 15 \mathrm{~min}\right)$ and removed in an acetic acid bath for $8 \mathrm{~min}$. An oxygen plasma treatment was performed to cross-link the PS chains and remove the RCP at the bottom of the pores. In the case of the lamellar features, the acetic acid bath was not performed to prevent the collapse of the whole structure. The removal of the PMMA component has been performed by oxygen plasma treatment. The morphology of the DBC films was characterized by Scanning Electron Microscopy (SEM) images (Zeiss Supra 40 SEM).

\subsection{Thermal annealing in RTP}

Similarly to the standard thermal annealing performed in furnace or hotplate the annealing process in RTP is divided in three phases (see figure 1): a) the heating ramp, b) the plateau and c) the relaxation ramp. The heating ramp (figure 1a) of all the samples described in this work is set constant at $18^{\circ} \mathrm{C} / \mathrm{s}$. This rate can be easily traced out by a thermocouple. In order to adapt the use of RTP to thermal treatment, characterized by short plateau and temperature relatively low compared to the common ones of semiconductor processes, it is necessary to set an overheating in the nominal temperature. This procedure governs the radiative power of halogen lamps making stable the measured temperature, which finally results in an effective plateau (figure 1b). The process ends with a relaxation ramp (figure 1c), whose velocity can be tuned by changing the flow rate of the $\mathrm{N}_{2}$ flux. The time in which the temperature is brought under the $\mathrm{T}_{\mathrm{g}}$ with a fixed $\mathrm{N}_{2}$ flux of $1000 \mathrm{sccm}$ at $\mathrm{T}=250^{\circ} \mathrm{C}$ is about $20 \mathrm{~s}$. Depending on heating rate, plateau duration and relaxation ramp it is possible to precisely control the time interval in which BCP remains above $\mathrm{T}_{\mathrm{g}}$. It is worth to remark 
that, since the heating and relaxation ramp are the same for all the processes, in the following the thermal treatments of the samples are labeled with the temporal duration of the plateau.

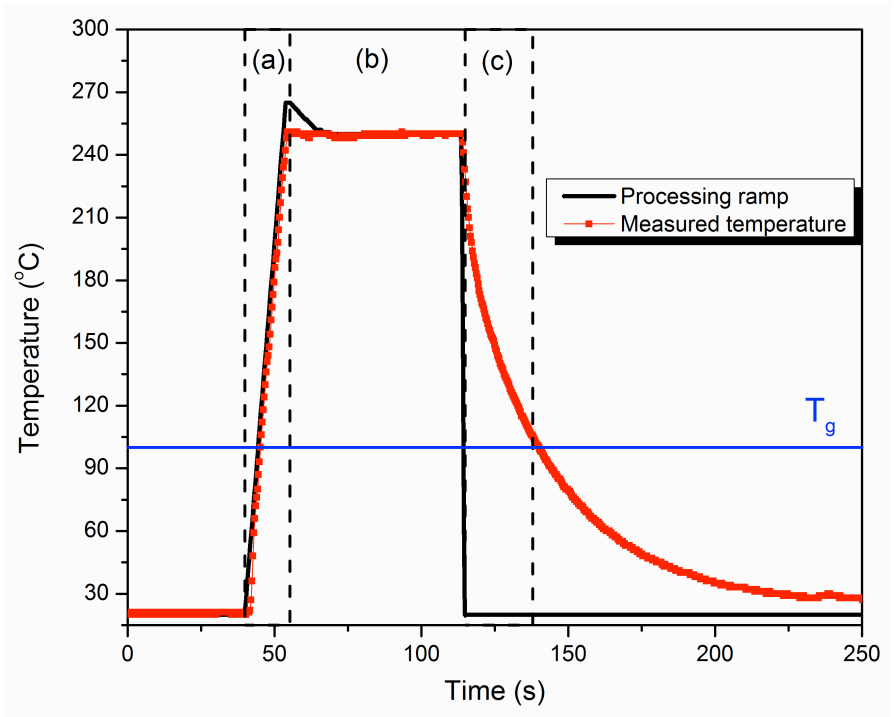

Figure 1. Scheme of the RTP annealing performed: a) Heating ramp brings BCP above $\mathrm{T}_{\mathrm{g}}$, up to temperature just below $\mathrm{T}_{\text {odt }}$ b) A nominal overheating of halogen lamps allows keeping constant the measured temperature for the duration of the chosen plateau. c) The relaxation ramp is influenced by the proper time of cooling of RTP.

\subsection{Calculation of the correlation length}

The determination of the correlation length on the lamellar samples was performed following the procedure described by Harrison et al. ${ }^{[22]}$ Several SEM images were analyzed for each sample. In order to eliminate eventual size effects, the lateral dimension of the images was taken at least 15 times larger than the correlation length. Furthermore, the resolution of the SEM images was set to obtain at least 30 pixels in a full period of the striped pattern $(\approx 0.9 \mathrm{~nm} / \mathrm{pixel})$.

\subsection{Determination of toluene content}

The residual toluene in the films was determined by Gas Chromatography-Mass Spectrometry (GCMS) technique. The samples were subjected to a solvent extraction performed according to the procedure reported in Ref. [23], using dichloromethane as the solvent. Each coated wafer was let to stand in contact with $1.0 \mathrm{~mL}$ of dichloromethane for $30 \mathrm{~min}$. Then, the dichloromethane was replaced with another aliquot of $1.0 \mathrm{~mL}$ of fresh dichloromethane and, after $30 \mathrm{~min}$ extraction time, the two aliquots were combined and the final solution filtered. $5.0 \mu \mathrm{L}$ of this solution were injected in the gas chromatograph Thermo GC-MS. The GC separation was carried out using a Phenomenex DB5-5ms capillary column (30 m, 0.25 i.d., 0.25 thickness). Oven temperature heating rate was 
$10^{\circ} \mathrm{C} / \mathrm{min}$ from 45 to $110^{\circ} \mathrm{C}$ and $60^{\circ} \mathrm{C} / \mathrm{min}$ from 110 to $180^{\circ} \mathrm{C}$. The injector temperature was $250^{\circ} \mathrm{C}$ in split mode with a split ratio of 1:10 and the carrier gas was helium at constant flow $(1.0 \mathrm{~mL} / \mathrm{min})$. The transfer line temperature was set at $280^{\circ} \mathrm{C}$.

The MS signal was acquired in EI+ mode with an Ionization Energy of $70.0 \mathrm{eV}$ and the ion source temperature was $250^{\circ} \mathrm{C}$. The acquisition was performed both in full-scan mode, in the $20-450 \mathrm{~m} / \mathrm{z}$ range, and in Single Ion Monitoring (SIM) acquisition mode at $92 \mathrm{~m} / \mathrm{z}$. The SIM signal was used in the quantification process by the integration of the relative chromatographic peak, identified by comparison with the standard retention time and mass spectrum. The quantification was carried out by performing a toluene calibration curve in the suitable concentration range.

\section{Results and discussion}

To elucidate the SA evolution during the RTP annealing, two set of samples were prepared by spincasting toluene solutions, containing either an asymmetric PS-b-PMMA (styrene unit fraction 0.71) or a symmetric PS-b-PMMA (styrene unit fraction 0.50 ), on silicon substrates properly neutralized with PS-r-PMMA random copolymers. To promote self-organization of the former DBC films into the cylinder phase, consisting of PMMA cylinders in the PS matrix, the standard annealing process in furnace requires a temperature of $190^{\circ} \mathrm{C}$ maintained constant for 2 hours. For the latter DBC, the perpendicularly oriented lamellar phase was achieved by annealing in furnace for 32 hours at $190^{\circ} \mathrm{C}$. These samples will be taken as reference for the system considered at the thermodynamic equilibrium. The samples annealed in the RTP were thermally treated at $190^{\circ} \mathrm{C}$ and $250^{\circ} \mathrm{C}$ for time periods ranging from 10 to $900 \mathrm{~s}$.

The morphological evolution of the films of both symmetric and asymmetric PS-b-PMMA, subjected to different thermal treatments, was investigated by SEM. Figure 2 illustrates the effect of RTP annealing at $190^{\circ} \mathrm{C}$. For comparison, the same figure reports the SEM images of samples prepared by the furnace process (figures $2 \mathrm{a}$ and $2 \mathrm{e}$ ). After $10 \mathrm{~s}$ of annealing time (figures $2 \mathrm{~d}$ and $2 \mathrm{~h}$ ), the morphological organization is at a very preliminary stage. Neither typical lamellar pattern nor standard hexagonal distribution of cylinders can be clearly observed. However, the perpendicular orientation of the microphase separated morphologies is already apparent, whereas extremely limited lateral ordering occurs. For annealing times longer than $60 \mathrm{~s}$ (figures $2 \mathrm{~b}-\mathrm{c}$ and $2 \mathrm{f}-$ $\mathrm{g}$ ), the lateral ordering is clearly improved although defective lamellar and cylindrical morphologies are obtained, even after $600 \mathrm{~s}$ annealing time. In summary, at $190^{\circ} \mathrm{C}$, phase separation and perpendicular ordering are easily obtained in a quite short time (annealing time $\leq 60 \mathrm{~s}$ ), but the lateral ordering process spans over longer time periods. 


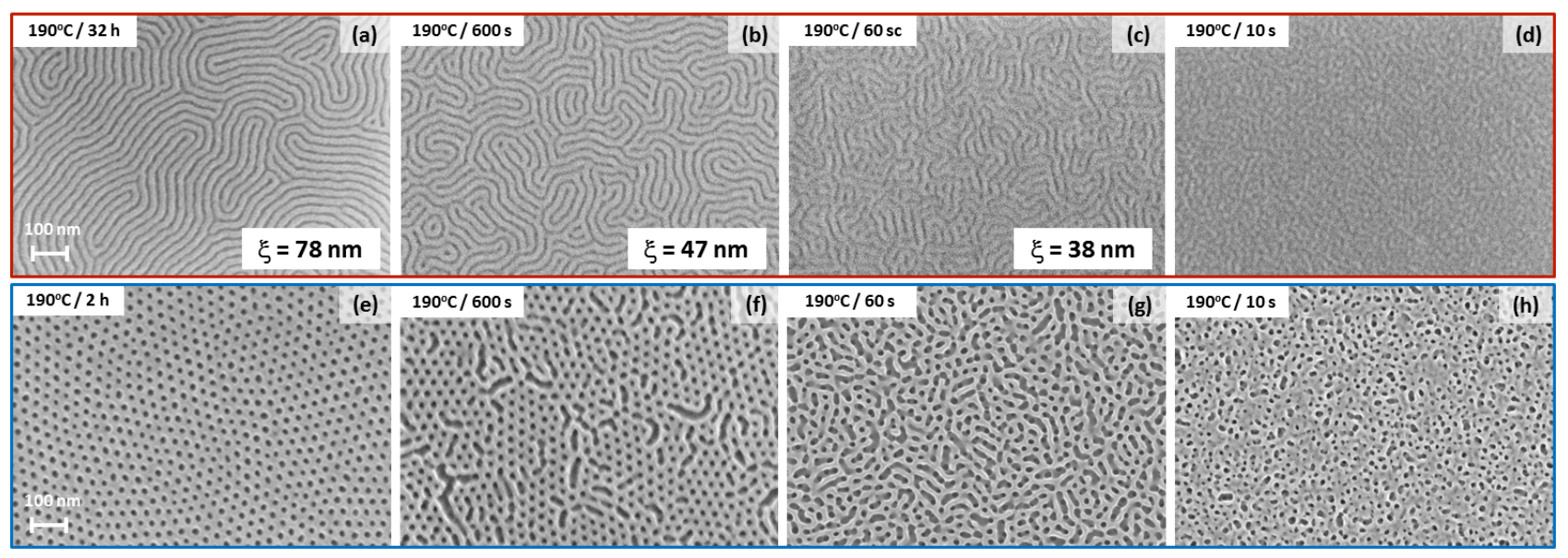

Figure 2. Morphology of lamellar and cylindrical samples annealed at $190^{\circ} \mathrm{C}$ in furnace (a, e) and in RTP (lamellar b-d and cylindrical f-h) for different time periods.

A semi-quantitative description of the morphological evolution with time can be obtained from the determination of the correlation length $(\xi)^{[24]}$ in the lamellar samples. The correlation length increases from $\xi=38 \mathrm{~nm}$ after $60 \mathrm{~s}$ to $\xi=47 \mathrm{~nm}$ after $600 \mathrm{~s}$. Although the RTP process allows the DBC self-assembly to be obtained in a very short time, the maximum achievable $\xi$ is lower than the one of the samples prepared in the furnace process at the same temperature (i.e. $\xi=78 \mathrm{~nm}$ reported in figure 2a). Surprisingly the latter value is significantly higher than those reported in literature for DBCs with the same molecular weight. ${ }^{[25]}$ The reason for this discrepancy is not clear at the moment although we could speculate about the processing conditions of the polymeric film in vacuum. In a previous paper we demonstrated that variation of the partial pressure during the thermal treatment strongly affect the final arrangement of the block copolymer. ${ }^{[26]}$ Nevertheless the $\xi$ values obtained after a $10 \mathrm{~min}$ thermal treatment in RTP are equivalent, within the experimental error, to those reported for the same block copolymer after $100 \mathrm{~min}$ long annealing at $190^{\circ} \mathrm{C}$ in vacuum. ${ }^{[25]}$

One simple method in order to increase the correlation length is to rise the annealing temperature. ${ }^{[21]}$ This approach has been demonstrated to be effective on prepatterned substrates although limited reduction of the local defectivity was achieved for block copolymers deposited on unpatterned surfaces, due to the lengthy processing times of perpendicularly oriented symmetric DBC ${ }^{[19]}$ Considering that the estimated bulk $\mathrm{T}_{\text {odt }}$ for PS-b-PMMA with $\mathrm{M}_{\mathrm{w}}>35000$ is higher than $280{ }^{\circ} \mathrm{C}^{[27]}$ and that the $\mathrm{T}_{\text {odt }}$ further rises in nanometric films, ${ }^{[21]}$ a second set of samples was RTP treated at $250^{\circ} \mathrm{C}$ and the morphological evolution as a function of time is illustrated in figure 3. Even at this temperature, the perpendicular ordering of lamellar and cylindrical domains is obtained 
after a very short annealing time $(\mathrm{t}<10 \mathrm{~s})$. In addition, the lateral ordering is quite high even after only $10 \mathrm{~s}$.

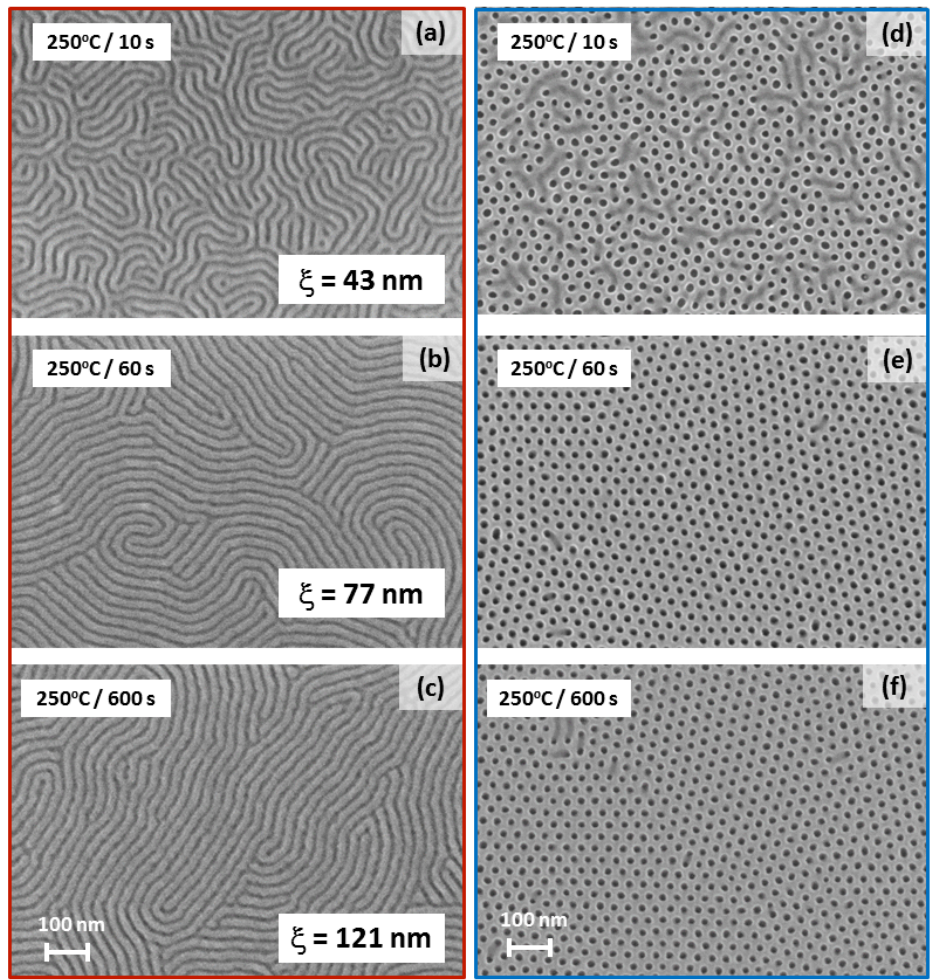

Figure 3. Morphology of lamellar (a-c) and cylindrical (d-f) samples annealed at $250^{\circ} \mathrm{C}$ for different time periods.

The correlation length of the lamellar samples annealed at $250^{\circ} \mathrm{C}$ was measured and data are reported in figure 4 as a function of the annealing time. After $300 \mathrm{~s}$ annealing time, a correlation length of $120 \mathrm{~nm}$ is obtained, which is much higher than the values currently reported in the literature $\left(\xi \sim 60 \mathrm{~nm}\right.$ at $250^{\circ} \mathrm{C}$ for 100 minutes of annealing time in vacuum). ${ }^{[25]}$ Moreover the $\xi$ values are significantly higher than those we obtained at $190^{\circ} \mathrm{C}$ in RTP and in furnace. This result is somehow surprising since several papers reported that the $\xi$ values exhibit limited temperature dependence for the symmetric PS-b-PMMA system on unpatterned substrates. ${ }^{[25,28]}$ In particular Ruiz et al. ${ }^{[25]}$ reported a variation of $\xi$ values from 42 to $64 \mathrm{~nm}$ for annealing temperature ranging from $180^{\circ} \mathrm{C}$ and $285^{\circ} \mathrm{C}$ with a fixed annealing time of 100 min. On the contrary Welander et al. reported a significant improvement of the ordering of the block copolymer thin film deposited on patterned surfaces when increasing the temperature from $190^{\circ} \mathrm{C}$ to $250^{\circ} \mathrm{C}^{[21]}$ Similarly, authors demonstrated the possibility of improve the coarsening kinetics of lamellar films using a multilayer induced ordering approach. ${ }^{[29]}$ Considering the evolution of the correlation length with time, we observe that $\xi$ increases steeply at first, according to a first order exponential with a growth 
exponent $\phi$ of 0.29 . After $300 \mathrm{~s}$ annealing time, an abrupt decrease of the growth exponent takes place, which reduces to $\phi=0.05$, thus possibly indicating the occurrence of a dual lateral ordering regime. The data available in the literature are limited to the time scale $\mathrm{t}>300 \mathrm{~s}$ corresponding to the shadowed area in figure 4 . In this temporal frame a very slow coarsening of PS-b-PMMA lamellar phase striped patterns is reported, with growth exponent $\phi<0.1$ even for small PS-bPMMA molecules. ${ }^{[28]}$ On the contrary the $\phi$ value we obtained for $\mathrm{t}<300 \mathrm{~s}$ is considerably higher than the growth exponent for lamellar phase DBC with similar $\mathrm{M}_{\mathrm{n}}$ treated in a standard furnace, and is comparable with the results obtained for cylindrical-phase DBC parallel to the substrate. ${ }^{[28,29]}$

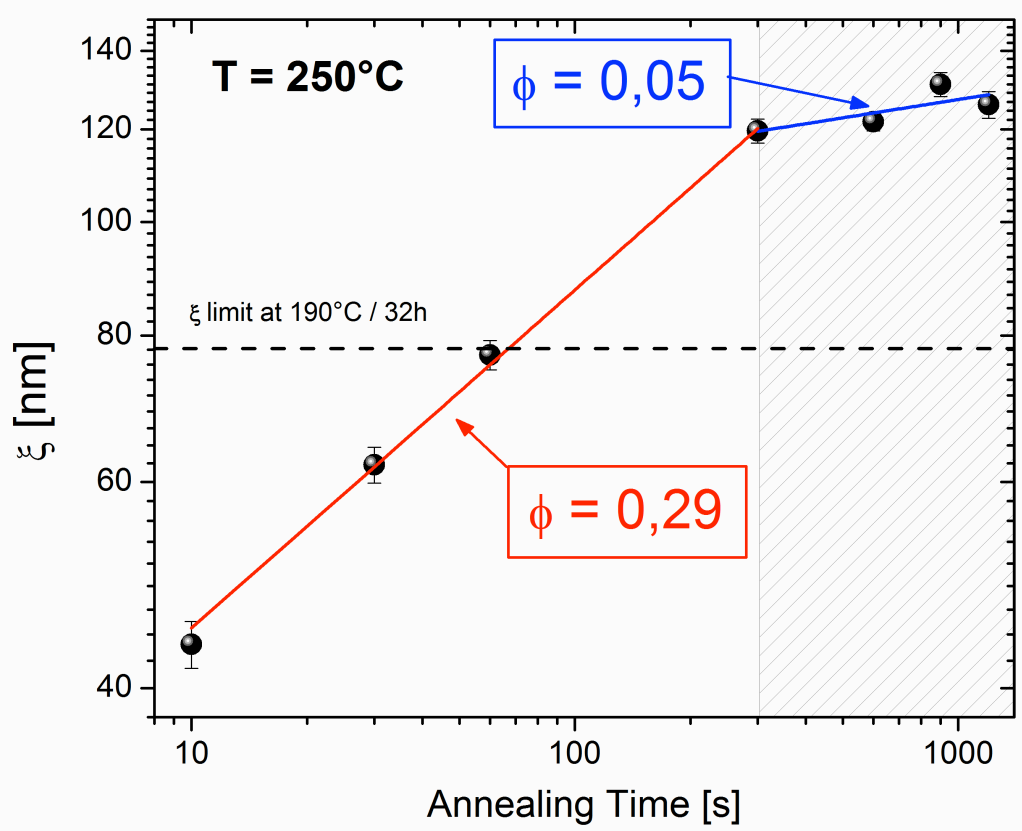

Figure 4. Correlation length $(\xi)$ of the lamellar samples as a function of the annealing time at $250^{\circ} \mathrm{C}$. Growth exponents $\phi$ of 0.29 and 0.05 are found by fitting the data with $\xi(t) \approx t$ before and after $300 \mathrm{~s}$ annealing time, respectively.

The experimental results we have collected so far demonstrate that the kinetics of the selfassembling of DBC during RTP thermal treatment is significantly enhanced compared to the data previously reported for conventional thermal treatments. The increased mobility of the polymeric chains could be ascribed to several factors. Among them the presence of the solvent retained in the nanometric film could play an important role in the morphological evolution of the DBC film. It is well know that solvent could be retained in thin polymeric films even after high temperature thermal treatment. ${ }^{[23]}$ The residual solvent in the film could result in a reduction of the $T_{g}$ and in a notable modification of the mobility of the polymeric chains. From GC-MS measurements, the amount of toluene trapped inside the spun films before and after the various RTP treatments was 
determined. The weights per unit area of toluene in the differently treated samples are collectively reported in figure 5, in which, for comparison purposes, also data relevant to the films obtained through the standard furnace process were included. The maximum amount of solvent is observed in the as-spun samples but is still quite high in the thermally treated ones.

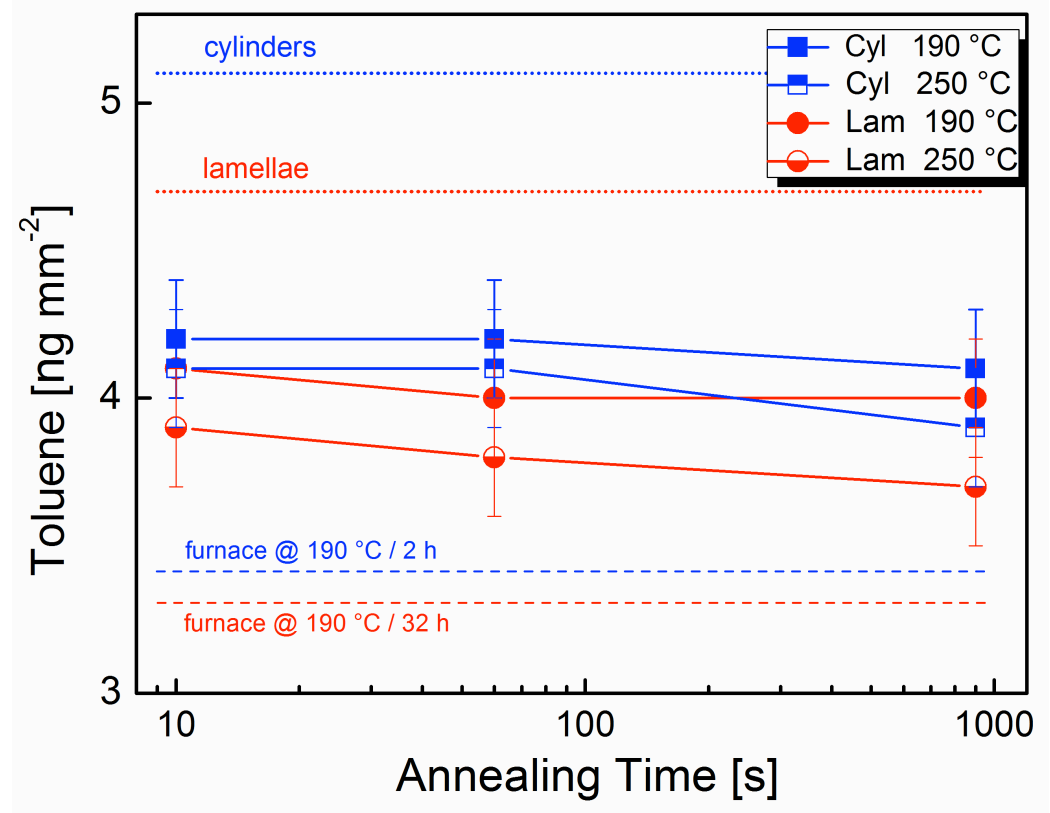

Figure 5. Toluene content in the DBC films subjected to various RTP treatments. The values concerning the as spun films and film annealed in furnace are also reported and represented by the blue and red dotted lines in the upper part of the graphic.

Films treated in furnace present toluene amounts significantly lower than those processed in RTP films. The RTP annealed samples exhibit a substantial reduction of the solvent content with respect to the as-spun samples although no further significant reduction of the solvent content is observed as a function of the annealing time in the timeframe 10-900 s. It is worth to note that asymmetric block-copolymers exhibit a higher solvent content than symmetric ones. This is consistent with the idea that toluene is a preferential solvent for PS fraction. The overall picture of these data demonstrates the significant solvent retention in ultrathin polymeric films. Literature data further suggest that the solvent is not uniformly distributed in the film but mainly located at the interface with the substrate. ${ }^{[23]}$ Consequently, the solvent retention is attributed to the combined effect of attractive interactions of the solvent with the substrate and a diminished solvent mobility in ultrathin films. In the present system, the decreased solvent mobility could result from the presence of hard walls, including the substrate and the brush structure of the random copolymer, which should reduce the number of configurations accessible to the block copolymer chains in the vicinity 
of the wall. The presence of solvent molecules at the interface should decrease this effect, thus decreasing the propensity of the solvent molecules to desorb.

In any case, data in Figure 5 indicate that, in the RTP process, a very fast solvent vaporization occurs during the $10 \mathrm{~s}$ annealing process, while a very slow solvent desorption takes place after the first $10 \mathrm{~s}$ of annealing. Solvent evaporation is known to be a strong driving force to promote selfassembling and ordering of block copolymer thin films. ${ }^{[30]}$ In particular the fast solvent vaporization provides a powerful and highly directional field able to promote perpendicular orientation of the microphase separated morphologies. This suggests that a clear picture of the self-assembling process in thin films during the RTP process requires a systematic and detailed study of the early stages $(\mathrm{t}<10 \mathrm{~s})$ of the annealing treatment. For these very short annealing times the effect of different heating and cooling rates on the residual solvent content has to be taken into account as well.

For annealing time $\mathrm{t}>10 \mathrm{~s}$ the retained solvent in the film is roughly stable, consequently the principal parameter acting on the ordering dynamics of the nanostructures is the annealing temperature; higher the annealing temperature higher the correlation length in the lamellar block copolymer thin film. This experimental evidence is consistent with previous observations reported by several groups in the literature. ${ }^{[21,25,28]}$ Nevertheless all the reports available in the literature indicate that in symmetric PS-b-PMMA block-copolymers higher temperatures only slightly increase the orientational order, with a large number of residual defects such as dislocations, disclinations, spirals and targets in the final polymeric pattern. In order to decrease the local defectivity of the DBC thin film and to increase the $\xi$ value, a pre-patterning of the substrate is necessary in order to guide the self-assembling of the PS-b-PMMA block copolymer. ${ }^{[25]}$ As previously discussed, the correlation length we obtained at $190^{\circ} \mathrm{C}$ after $60 \mathrm{~s}$ of annealing are comparable to those reported in the literature after much longer thermal treatment performed in vacuum at the same temperature. Moreover at $250^{\circ} \mathrm{C}$ we were able to achieve very high $\xi$ values without any pre-patterning of the substrate. Although a direct comparison is difficult due to the lack of information in the literature about the solvent content, we tentatively attribute this enhanced ordering of the polymeric film to the presence of the residual solvent that increases the plasticity of the block copolymer and increases the mobility of the macromolecules. The limited reduction of the solvent content when increasing the annealing temperature from $190^{\circ} \mathrm{C}$ to $250^{\circ}$ prevents any reduction in chain mobility that could go against the speed up of the process due to the increased temperature. Our data suggest an annealing mechanism in which both a solvent enriched environment and elevated temperature play a role. 
Even if an exhaustive explanation of the reduced local defectivity in the self-assembled blockcopolymers after the RTP process has not been produced yet, it is quite clear that this peculiar thermal treatment offers the possibility to promote the self-assembling of polymeric film with reduced defectivity in a very short time. In conventional thermal treatment the orientational correlation of symmetric block copolymer thin film evolves much more slowly than in asymmetric films, limiting their use in applications that require a long-range orientational order. The proposed approach allows removing this important limitation that prevent the technological exploitation of symmetric block copolymers without introducing any pre-patterning of the substrate. Moreover the RTP process provides the unique opportunity to systematically investigate the self assembling of the block copolymer in a time frame $(\mathrm{t}<60 \mathrm{~s})$ that is not accessible using conventional hot-plates and furnaces. Block copolymer self-assembling in $60 \mathrm{~s}$ has already been demonstrated for lamellar PS-b-PMMA block copolymers on pre-patterned substrates using hot plates. ${ }^{[21]}$ Yet the investigation of the early stages $(\mathrm{t}<60 \mathrm{~s})$ of block copolymer self assembly using hot plates is prevented by the conduction mechanism that govern the heating of the sample in contact with the hot plate. The use of radiation sources in RTP allows controlling the heating transfer to the polymeric film in a very precise way, determining the effective thermal budget of the process.

\section{Conclusions}

In conclusion, we have demonstrated that RTP, via the the extremely fast temperature modulation above and below the glass transition, represents an extremely efficient method to induce selforganization in DBC thin films, in time periods well below the $240 \mathrm{~s}$ limit defined by the ITRS. In fact, the formation of well-ordered cylindrical and lamellar features, perpendicularly oriented with respect to the substrate, was obtained in less than $60 \mathrm{~s}$ at $250^{\circ} \mathrm{C}$. The proposed methodology allowed unveiling the DBC ordering dynamics in the time range $\mathrm{t}<60 \mathrm{~s}$. The analysis revealed an unexpected fast evolution of perpendicularly oriented symmetric block copolymer during the early stages of the self-assembling process. Moreover, increasing the annealing temperature to $250^{\circ} \mathrm{C}$, significant reduction of the defectivity in symmetric PS-b-PMMA block copolymer was achieved in a very short time without any pre-patterning of the substrate. Finally it is worth to remind that this technology can be fully integrated in the standard process flow of semiconductor industry, thus allowing DBCs to exploit their full potential as real alternatives to the current lithographic processes. 


\section{Acknowledgments}

This research activity was financially supported by the NANO-BLOCK project ("NANO-device fabrication using Block copolymer based technology"). Patent protection related to this work is pending.

\section{References}

1. Y.H. Wang, C.A. Mirkin, S-J. Park, ACS Nano 2009, 3, 1049-1056.

2. H.M. Saavedra, T.J. Mulen, P. Zhang, D.C. Dewey, S.A. Claridge, P.S. Weiss, Rep. Prog. Phys. 2010, 73, 036501.

3. J.Y. Cheng, C.A. Ross, H.I. Smith, E.L. Thomas, Adv. Mat. 2006, 18, 2505-2521.

4. R.F. Pease, S.Y. Chou, Proceedings of the IEEE 2008, 96, 248-270.

5. M. Lazzari, M.A. Lopez-Quintela, Adv. Mater. 2003, 15, 1583-1594.

6. H. Yi, X-Y. Bao, J. Zhang, C. Bencher, L-W. Chang, X. Chen, R. Tiberio, J. Conway, H. Dai, Y. Chen, S. Mitra, H.-S. P. Wong, Adv. Mater. 2012, 24, 3107-3114.

7. M. Park, C. Harrison, P.M. Chaikin, R.A. Register, D.H. Adamson, Science 1997, 276, 1401-1404.

8. J. Bang, U. Jeong, D.Y. Ryu, T.P. Russel, C.J. Hawker, Adv. Mat. 2009, 21, 4769-4792.

9. The International Technology Roadmap for Semiconductors (ITRS), "Emerging Research Materials", 2011.

10. A. Andreozzi, E. Poliani, G. Seguini, M. Perego, Nanotechnology 2011, 22, 185304.

11. X.M. Yang, L. Wan, S. Xiao, Y. Xu, D.K. Weller, ACS Nano 2009, 3, 1844-1858.

12. R. Ruiz, H. Kang, F.A. Detcheverry, E. Dobisz, D.S. Kercher, T.R. Albrecht, J.J. de Pablo, P.F. Nealey, Science 2008, 321, 936-939.

13. D.Y. Ryu, S. Ham, E. Kim, U. Jeong, C.J. Hawker, T.P. Russel, Macromolecules 2009, 42, 4902-4906.

14. E. Han, K.O. Stuen, Y-H. La, P.F. Nealey, P. Gopalan, Macromolecules 2008, 41, 90909097.

15. The International Technology Roadmap for Semiconductors (ITRS), "Emerging Research Materials", 2007.

16. K.W. Gotrik, A.F. Hannon, J.G. Son, B. Keller, A. Alexander-Katz, C.A. Ross, ACS Nano 2012, 6, 8052-8059.

17. J.G. Son, J-B. Chang, K.K. Berggren, C.A. Ross, Nano Lett. 2011, 11, 5079-5084. 
18. J. Gong, H. Ahn, E. Kim, H. Lee, S. Park, M. Lee, S. Lee, T. Kim, E.-A. Kwak, Du Yeol Ryu, Soft. Matter 2012, 8, 3570-3575.

19. X. Zhang, K.D. Harris, N.L.Y. Wu, J.N. Murphy, J.M. Buriak, ACS Nano 2010, 4, 70217029.

20. K.W. Guarini, C.T. Black, S.H.I Yeung, Adv. Mat. 2002, 14, 1290-1294.

21. A.M. Welander, H. Kang, K.O. Stuen, H.H. Solak, M. Muller, J.J. de Pablo, P.F. Nealey, Macromolecules 2008, 41, 2759-2761.

22. C. Harrison, P.M. Chaikin, D.A. Huse, R.A. Register, D.H. Adamson, A. Daniel, E. Huang, P. Mansky, T.P. Russel, C.J. Hawker, D.A. Egolf, I.V. Melnikov, E. Bodenschatz, Macromolecules 2000, 33, 857-865.

23. J. García-Turiel, B. Jérôme, Colloid Polym. Sci. 2007, 285, 1617-1623.

24. C. Harrison, Z. Cheng, S. Sethuraman, D.A. Huse, P.M. Chaikin, D.A. Vega, J.M. Sebastian, R.A. Register, D.H.Adamson, Phys. Rev. E, 2002, 66, 011706.

25. R. Ruiz, N. Ruiz, Y. Zhang, R.L. Sandstorm, C.T. Black, Adv. Mat. 2007, 19, 2157-2162.

26. I. A. Zucchi, E. Poliani, M. Perego, Nanotechnology 2010, 21, 185304.

27. H. Ahn, D.Y. Ryu, Y. Kim, K.W. Kwon, J. Lee, J. Cho, Macromolecules 2009, 42, $7897-$ 7902.

28. R. Ruiz, J.K. Bosworth, C.T. Black, Phys Rev B 2008, 77, 054204.

29. R. Ruiz, R.L. Sandstorm, C.T. Black, Adv. Mat. 2007, 19, 687-691.

30. S.H. Kim, M.J. Misner, T. Xu, M. Kimura, T.P. Russell, Adv. Mat. 2004, 16, 226-231. 